\title{
A NOTE ON CYCLIC ALGEBRAS OF ORDER SIXTEEN
}

\section{BY A. A. ALBERT*}

1. Introduction. In a recent paper $\dagger$ I considered cyclic (Dickson) algebras of order sixteen generated by a cyclic quartic field $Z$ and a quantity $\gamma$ in the reference field $F$. It was proved there that if the algebra $A$ were a division algebra and if $\gamma^{2}$ were the norm of a quantity of $Z$, so that the Wedderburn norm condition would not be satisfied, then $A$ would be the direct product of two generalized quaternion algebras. It was not proved, however, that such division algebras existed.

$R$. Brauer has recently $\ddagger$ proved that there exist normal division algebras which are direct products of two generalized quaternion algebras. In the present note I give an example of a cyclic algebra over the Brauer reference field for which the norm condition is not satisfied, therefore completing the theory of the previous paper.

2. The Example. Let $F=R(\xi, \eta)$, where $\xi$ and $\eta$ are indeterminates and $R$ is the field of all rational numbers. This is the reference field of the algebras of Brauer. We shall use the notations of my paper (loc. cit.), Theorem 3. It was proved there that a necessary and sufficient condition that a direct product of two generalized quaternion algebras over $F$ be a division algebra is that the connected form

$$
\tau x_{1}^{2}+\sigma x_{2}^{2}-\sigma \tau x_{3}^{2}-\left(\gamma x_{4}^{2}+\rho x_{5}^{2}-\rho \gamma x_{6}^{2}\right),
$$

in the variables $x_{1}, \cdots, x_{6}$ in $F$ be not a null form. We shall take

$$
\sigma=-2 \xi^{3}, \rho=\eta, \gamma=-1, \tau=\alpha,
$$

where $\alpha$ is a rational number not the square of a rational number.

Suppose that $\alpha=\nu^{2}$, where $\nu$ is in $F$. Then we may write

$$
\nu=b c^{-1} \text {, }
$$

\footnotetext{
* Presented to the Society, September 9, 1931.

$\dagger$ This Bulletin, vol. 37 (1931), pp. 301-312.

‡ Mathematische Zeitschrift, vol. 31 (1930), §5. Brauer's example is that of an algebra not necessarily a cyclic algebra and it would probably be difficult to prove it cyclic even if this were the case. Also his proof that the algebra is a division algebra is essentially different from ours.
} 
where $b=b(\xi, \eta)$ and $c=c(\xi, \eta)$ are polynomials in $\xi$ and $\eta$ with coefficients in $R$ such that the greatest common divisors of $b$ and $c$ are rational numbers. Then

$$
b^{2}=\alpha c^{2}, \quad b_{1}^{2}=\alpha c_{1}^{2},
$$

where $b_{1}=b(\xi, 0), c_{1}=c(\xi, 0)$. The coefficient of the highest power of $\xi$ in $b_{1}^{2}$ is evidently a rational square, that in $c_{1}^{2} \alpha$ not such a square, since $\alpha$ is not a rational square. Hence $b_{1}{ }^{2}=c_{1}{ }^{2} \alpha$ implies that $b_{1}=c_{1}=0$ and $b$ and $c$ have the common factor $\eta$, a contradiction.

Suppose that (1) vanished for $x_{1}, \cdots, x_{6}$ not all zero and in $F$. Evidently we could take $x_{1}, \cdots, x_{6}$ to be polynomials in $\xi$ and $\eta$ with rational coefficients and with greatest common divisor a rational number. We thus write

$$
x_{i}=x_{i}(\xi, \eta),
$$

with rational coefficients and having no factor in common. Equation (1) becomes

$$
\alpha x_{1}^{2}-2 \xi^{3} x_{2}^{2}+2 \alpha \xi^{3} x_{3}^{2}=-x_{4}^{2}+\eta\left(x_{5}^{2}+x_{6}^{2}\right),
$$

which must be satisfied identically in $\xi$ and $\eta$ so that it must be satisfied when we replace $\eta$ by zero. Let $x_{i}(\xi, 0)=y_{i}$ and let the highest power of $\xi$ occurring in $y_{i}$ be $\xi^{r_{i}}$, its coefficient being $\lambda_{i}$ where $\lambda_{i}=0$ if and only if $y_{i}=0$. Then (3) becomes

$$
y_{4}^{2}+\alpha y_{1}^{2}=2 \xi^{3}\left(y_{2}^{2}-\alpha y_{3}^{2}\right),
$$

identically in $\xi$. According as $r_{4}>r_{1}, r_{4}<r_{1}, r_{4}=r_{1}$, the term of highest degree in $y_{4}{ }^{2}+\alpha y_{1}{ }^{2}$ is

$$
\lambda_{4}^{2} \xi^{2 r_{4}}, \alpha \lambda_{1}^{2} \xi^{2 r_{1}},\left(\lambda_{4}^{2}+\alpha \lambda_{1}^{2}\right) \xi^{2 r_{1}},
$$

an even power, and is zero if and only if $y_{1}=y_{4}=0$. Similarly the possible terms of highest degree in $2 \xi^{3}\left(y_{2}{ }^{2}-\alpha y_{3}{ }^{2}\right)$ are

$$
2 \lambda_{2}{ }^{2} \xi^{2 r_{2}+3},-2 \alpha \lambda_{3}{ }^{2} \xi^{2 r_{3}+3}, 2\left(\lambda_{2}{ }^{2}-\alpha \lambda_{3}{ }^{2}\right) \xi^{2 r_{2}+3},
$$

so that (3) and its consequence (4) imply that a polynomial whose degree is even is equal to a polynomial whose degree is odd. This is possible only when both polynomials are zero, so that $\lambda_{4}=\lambda_{1}=\lambda_{2}=\lambda_{3}=0$ and hence $y_{1}=y_{2}=y_{3}=y_{4}=0$. But then $x_{1}, \cdots, x_{4}$ are divisible by $\eta$, so that we may write 


$$
x_{i}=\eta z_{i}, \quad(i=1, \cdots, 4)
$$

and obtain

$$
\eta^{2}\left(z_{1}^{2}-2 \xi^{3} z_{2}^{2}+2 \alpha z_{3}^{2}+z_{4}^{2}\right)=\eta\left(x_{5}^{2}+x_{6}^{2}\right),
$$

from (3). It follows that $x_{4}^{2}+x_{6}{ }^{2}$ is divisible by $\eta$. Let the constant term with respect to $\eta$ of $x_{5}$ be $\mu_{5}$, that of $x_{6}$ be $\mu_{6}$. Then obviously $\mu_{5}{ }^{2}+\mu_{6}{ }^{2}=0$, where $\mu_{5}$ and $\mu_{6}$ are polynomials in $\xi$ with rational coefficients. If $\mu_{5} \neq 0$, then $-1=\left(\mu_{6} \mu_{5}^{-1}\right)^{2}$, which is impossible as we have shown, since -1 is not a rational square. Hence $\mu_{5}=\mu_{6}=0$ and each of the quantities $x_{5}, x_{6}$ is divisible by $\eta$. But then $x_{1}, \cdots, x_{6}$ are all divisible by $\eta$, a contradiction of our hypothesis. Hence we have proved that (3) is not a null form, the direct product of our two generalized quaternion algebras

$$
\begin{aligned}
& B=(1, u, s, u s), \quad u s=-s u, \quad u^{2}=\tau, \quad s^{2}=\sigma, \\
& C=(1, j, t, j t), \quad t j=-j t, \quad j^{2}=\gamma, \quad t^{2}=\rho,
\end{aligned}
$$

is a division algebra.

We now take $\alpha=\tau=1+\Delta^{2}$, where $\Delta$ is a rational number so chosen that $1+\Delta^{2}$ is not a rational square. For example $\Delta$ may be taken to be unity. Let

$$
\beta_{1}=\xi, \quad \gamma_{0}=-\beta_{1}^{2}, \quad \nu=-\eta\left(2 \alpha \xi^{2}\right)^{-1} .
$$

The author has shown that the equation

$$
\phi(\omega) \equiv \omega^{4}+2 \nu\left(1+\Delta^{2}\right) \omega^{2}+\nu^{2} \Delta^{2}\left(1+\Delta^{2}\right)=0
$$

is a cyclic quartic over $F$ for every $\nu \neq 0$ in $F$ and $\tau=1+\Delta^{2}$ not the square of any quantity of $F$. Our above choices of $\nu$ and $\tau$ evidently insure that these requirements are satisfied. I have also shown (loc. cit.) that if we define the cyclic algebra with the basis

$$
x^{r} y^{s}, \quad(r, s=0,1,2,3),
$$

such that

$$
\phi(x)=0, \quad y^{r} x=\theta^{r}(x) y^{r}, \quad y^{4}=\gamma_{0}, \quad(r=0,1, \cdots),
$$

then, if $\gamma_{0}=-\beta_{1}{ }^{2}$, by Theorem 4 the algebra is expressible as the direct product of two generalized quaternion algebras $B$ and $C$ with 


$$
\tau=1+\Delta^{2}, \sigma=2 \beta_{1} \gamma_{0}, \rho=2 \nu \beta_{1} \tau\left(-\beta_{1}\right), \gamma=\gamma_{0}=-\beta_{1}{ }^{2} .
$$

But $2 \beta_{1} \gamma_{0}=-2 \xi^{3}, \rho=\eta$ and we may evidently choose a new basis with $\gamma=\gamma_{0}$ replaced by -1 . Hence $B \times C$ is a division algebra. Moreover $\gamma_{0}=-\beta_{1}{ }^{2}, \gamma_{0}{ }^{2}=\beta_{1}{ }^{4}$ is the norm of the scalar $\beta_{1}$ in $F(x)$. For this cyclic algebra the Wedderburn norm condition is not satisfied and yet the algebra is a division algebra.

The University of Chicago

\title{
AN ACKNOWLEDGMENT
}

\author{
BY G. W. STARCHER
}

Since the publication of my article entitled $A$ note on geometrical factorial series in the June issue of this Bulletin,* my attention has been called to the fact that most of the results of that paper are not new and have been previously given by F. Ryde. $\dagger$ Ryde considers a series which is essentially the same as (1) and calls it a "geometric factorial series." The term geometric factorial series was suggested to me by the fact that Cauchy had called the denominators of the terms in the series (1) geometric factorials. $\ddagger$ Later geometric was changed to geometrical.

The conclusions in $\$ 2$ are given by Ryde (page 6) and the comparison of (1) and (2) was evidently known to him. The theorem in $\$ 3$ is proved in essentially the same way by Ryde (pages 6-8), and on page 8 a more general expansion is given. On pages 11-13 he employs the multiplication of such series, and he probably had formulas for the coefficients in such a product.

OHIO UNIVERSITY

* This Bulletin, vol. 37 (1931), pp. 455-463.

$\dagger A$ contribution to the theory of linear homogeneous geometric difference equations ( $q$-difference equations), Lund dissertation, published by Lindstedts Univ. Bokhandel (1921).

$\ddagger$ Encyclopédie des Sciences Mathématiques, vol. I, 4, p. 279. 